\title{
Diagnosis of hepatoma using grayscale and Doppler ultrasound in patients with chronic liver disease
}

This article was published in the following Dove Press journal:

International Journal of General Medicine

28 October 201I

Number of times this article has been viewed

\section{Wasim A Memon \\ Zishan Haider \\ Mirza Amanullah Beg \\ Muhammad Idris \\ Tanveer-ul-Haq \\ Waseem Akhtar \\ Sidra Idris}

Radiology Department, Aga Khan University Hospital, Karachi, Pakistan

Every author contributed equally to the work
Correspondence: Zishan Haider Radiology Department, Aga Khan University Hospital, Stadium Road, Karachi, 74500 Pakistan

Tel +92 2I 34862020

Fax +92 21 34934294

Email zishan.haider@aku.edu
Objective: To determine the diagnostic accuracy of liver ultrasound for the detection of hepatoma in chronic liver disease (CLD) patients by either taking histopathology or serum $\alpha$-fetoprotein levels or a biphasic computed tomography (CT) scan (whichever is available) as the gold standard.

Study design: Cross-sectional.

Place and duration of study: Radiology Department, The Aga Khan University Hospital, Karachi, Pakistan, from January 2007 to January 2010.

Methods: A total of 239 patients (156 males and 83 females) with clinical suspicion or surveillance of hepatoma in CLD referred to the radiology department for ultrasound evaluation followed by either liver biopsy and histopathology or serum $\alpha$-fetoprotein level or biphasic CT scan.

Results: The sensitivity of ultrasound for hepatoma detection in CLD was 65\%, specificity was $85 \%$, and accuracy was $70 \%$, and positive predictive value and negative predictive value were $92 \%$ and $45 \%$, respectively.

Conclusion: Ultrasound is a relatively quick, safe, reasonably accurate, and noninvasive imaging modality for the detection of hepatoma in CLD and can be complemented with clinical assessment of screening high-risk patients.

Keywords: hepatoma, ultrasound, radiology, chronic liver disease

\section{Introduction}

Hepatocellular carcinoma (HCC) is the most common primary hepatic neoplasm that occurs in the setting of chronic liver disease (CLD) and cirrhosis. ${ }^{1}$ Ultrasound is the most commonly used imaging modality for screening CLD patients for the detection of tumor, because of its reliability, cost-effectiveness, and noninvasiveness. ${ }^{2}$ The incidence of HCC in the US is rising and has nearly doubled in the past 15 years because of an increasing incidence of hepatitis $\mathrm{B}$ and $\mathrm{C}$ infections. ${ }^{3}$ In Pakistan, there is an incidence of $8 / 100,000$ per annum ${ }^{4}$ and a prevalence of $3.7 \%,{ }^{5}$ but it varies in the international data up to $39 \% .^{6-8}$ The accurate detection of HCC is critical for treatment and management, especially in this modern era of improved surgical techniques, which include tumor resection, liver transplantation, transcatheter chemoembolization, and radiofrequency ablation. ${ }^{9-11}$ Although serum $\alpha$-fetoprotein levels are used to screen HCC in CLD patients, this test sometimes remains inconclusive; ${ }^{12-14}$ however, the cutoff value of $\alpha$-fetoprotein above $400-500 \mathrm{ng} / \mathrm{mL}$ is considered diagnostic for hepatoma in CLD patients. $^{15}$ 
Imaging has a very important role to play. Ultrasound is the most widely used imaging modality for HCC screening in CLD patients. The sensitivity of ultrasound in the detection of $\mathrm{HCC}$ ranges from $59 \%$ to $84 \%{ }^{16,17}$ with a specificity of $96 \% .{ }^{18}$ Biphasic computed tomography (CT) scanning has a high sensitivity for detecting hepatoma, and earlier arterial enhancement and venous washout is diagnostic of hepatoma. ${ }^{14}$ Liver damaged by cirrhosis has benign lesions with areas of fat replacement, fibrotic scars, necrotic areas, or arteriovascular malformations that can mimic HCC and create diagnostic problems. ${ }^{19}$ Recent developments in ultrasound technology, such as Doppler ultrasound, power Doppler ultrasound, and contrast material-enhanced ultrasound, have shown promise for better characterization of liver lesions in patients with CLD. ${ }^{20,21}$ The data regarding ultrasound evaluation of hepatoma in CLD are limited from underdeveloped countries like Pakistan. Therefore, the objective of this study was to evaluate the role of grayscale and Doppler ultrasound in the detection of hepatoma in patients with CLD.

\section{Material and methods}

The participants of the study were included from patients referred to the radiology department of The Aga Khan University Hospital, Karachi, Pakistan, for ultrasound evaluation of the liver from January 2007 to January 2010, followed by either liver biopsy or serum $\alpha$-fetoprotein level or biphasic CT scan. Patients already known to have hepatoma were excluded. Ultrasound diagnosis of HCC was made if any two of the following findings were seen in the liver:

- Lesions smaller than $3 \mathrm{~cm}$ in diameter that appeared hypoechoic without posterior enhancement

- Any size lesion showing a heterogeneous mixed echogenicity pattern

- Diffuse infiltrating lesion with irregular margins

- The presence of pseudocapsule in lesion

- Mass protruding beyond the surface of the liver

- Displacement or compression of intrahepatic blood vessels.

After at least 6 hours' fasting, patients went through the ultrasound with a 3-6 Mhz curvilinear probe in a supine and oblique position, using departmental protocol. All examinations were performed and interpreted by credentialed radiologists. Hard-copy images were also recorded for documentation. Data were entered and analyzed using SPSS (version 15, SPSS, Inc, Chicago, IL). Sensitivity, specificity, accuracy, and negative and positive predictive values of ultrasound for hepatoma were calculated using histopathologic findings.

\section{Results}

A total of 239 patients fulfilled the inclusion criteria of the study and were included. Ultrasonography of 239 consecutive patients was carried out in the ultrasound suite of the hospital's department of radiology. All the included patients were clinically suspected to have HCC by the referring gastroenterologist. All these patients were already diagnosed to have CLD. A total of $156(65 \%)$ patients among these were males and $83(35 \%)$ were females. A wide range of age was seen (between 31 years and 70 years). Out of these 239 patients, ultrasound identified hepatoma in 126 (53\%) patients. Out of these, 117 (93\%) were found to have hepatoma on histopathology or serum $\alpha$-fetoprotein level or a biphasic CT scan, whereas nine (7\%) patients were false positive. Amongst the false-positive cases, seven patients were found to have metastatic disease and two patients had hemangiomas. A heterogeneous appearance of the lesion with greater than $5 \mathrm{~cm}$ size was the most common finding of hepatoma on ultrasound. This was seen in 62 of 117 cases. The presence of pseudocapsule was a frequent finding in true positive cases and was seen in 50\% of the cases. Associated intrahepatic vascular displacement was found in $26 \%$ of the cases. A total of $113(47 \%)$ were found to be negative on ultrasound. Out of these, $62(55 \%)$ were found to have hepatoma. The rest of the patients (51 [45\%]) were found to have changes of CLD.

Based on these findings, the sensitivity of ultrasound was found to be $65 \%$, specificity $85 \%$, positive predictive value $92 \%$, negative predictive value $45 \%$, and accuracy $70 \%$ for diagnosing hepatoma in CLD patients.

\section{Discussion}

HCC is the most common primary liver cancer and usually occurs as a complication of CLD, most often arising in a cirrhotic liver. Therefore, accurate surveillance of patients with liver cirrhosis is of great clinical importance because they are at increased risk for hepatoma. Different clinical approaches are present for screening patients for hepatoma, including serum $\alpha$-fetoprotein, ultrasonography, and CT. Ultrasound is a relatively safe, cost-effective, and easily available approach. In this study of 239 biopsied liver lesions we found sensitivity of ultrasound to be $65 \%$, specificity $85 \%$, positive predictive value $92 \%$, negative predictive value $45 \%$, and accuracy $70 \%$ for identifying hepatoma in CLD patients. These data are also 
comparable with the international data. The international data show sensitivity of $59 \% 0^{16,17}$ and a specificity of $96 \% .{ }^{17}$ In this study we have found $78(66.6 \%)$ cases of HCC with HCV, 26 $(22.2 \%)$ cases with HBV positivity, three patients (2\%) with both hepatitis B and C, seven (5.9\%) patients with hepatitis $\mathrm{B}$ and $\mathrm{D}$, and three ( $2 \%$ ) patients with alcoholic cirrhosis. Similar results have been shown in studies from all over Pakistan. Farooqi and Farooqi ${ }^{22}$ found $67.86 \%$ cases to be positive for the hepatitis $\mathrm{C}$ virus and 14\% with the hepatitis $\mathrm{B}$ virus. In a study from Lahore, around two-thirds of patients of $\mathrm{HCC}$ were seropositive for $\mathrm{HCV}$, and the remaining cases were due to the hepatitis $\mathrm{B}$ virus alone or a coinfection with both viruses. ${ }^{23}$ The most common presentation of HCC on ultrasound in our study was of a heterogeneous lesion of mixed echogenicity pattern (53\%) with size more than $5 \mathrm{~cm}$, which is comparable with a study by Koteish and Thuluvath. ${ }^{17}$ The other patterns found in our studies were lesions less than $3 \mathrm{~cm}$ in size with a hypoechoic appearance on ultrasound (28\%) and diffusely infiltrating hepatomas (19\%). Associated findings with these lesions were intrahepatic venous thrombosis, which was seen in $26 \%$ cases of hepatoma, mass protruding beyond the surface of the liver in $9 \%$ cases, and displacement of intrahepatic blood vessels in $6 \%$ of the cases. The most common disease in our study that was confused for hepatoma in CLD patient was metastatic lesions in seven (6\%) of 126 total positive cases on ultrasound. The other disease that was confused for hepatoma was hemangiomas in two ( $2 \%$ ) of 126 total positive cases on ultrasound.

The reason for diagnostic difficulty was the heterogeneity of these lesions on ultrasound. As the heterogeneous appearance was the most common presentation of hepatoma on ultrasound, any heterogeneous lesion in CLD patients should be evaluated very cautiously. Because ultrasound is a quick, cost-effective, easily available tool, it can be used as an immediate modality for evaluation of patients with CLD, especially in underdeveloped countries where affordability is a major issue and CT scans are not widely available. Our study has few limitations besides the single center and small sample size. A degree of experience among the radiologists in interpretations of ultrasound was not taken into account. Interobserver variability of ultrasound findings and patient-related factors were not assessed.

\section{Conclusion}

Ultrasound is a quick and cost-effective tool for the detection of hepatoma in clinically suspected cases and can be used as a screening tool in patients with CLD for surveillance of HCC.

\section{Disclosure}

No funding or any other financial support was used for this study.

\section{References}

1. Simonetti RG, Camma C, Fiorello F. Hepatocellular carcinoma: a world-wide problem and the major risk factors. Dig Dis Sci. 1991;36: 962-972.

2. Morimoto Y, Kubo S, Shuto T, et al. Power Doppler ultrasonographic diagnosis of small hepatocellular carcinomas. Dig Surg. 2002;19: 379-387.

3. El-Serag HB, Mason AC. Rising incidence of hepatocellular carcinoma in the United States. N Engl J Med. 1999;340:745-750.

4. Abdul Mujeeb S, Jamal Q, Khanani R, et al. Prevalence of hepatitis B surface antigen and $\mathrm{HCV}$ antibodies in hepatocellular carcinoma cases in Karachi, Pakistan. Trop Doct. 1997;27:45-46.

5. Jamal Q, Jaffarey NA, Aslam SM. A review of unusual liver tumors. J Pak Med Assoc. 1989;39:53-56.

6. Farooqi JI, Farooqi RJ. Prevalence of hepatocellular carcinoma in Pakistan in liver cirrhosis: an experience in NWFP. J Coll Physicians Surg Pak. 2000;2:54-55.

7. Zahir N, Mubarik A, Abdullah P. Spectrum of histopathological lesions in liver biopsies at PNS Shifa, Karachi. J Coll Physicians Surg Pak. $1998 ; 6: 255-257$.

8. Riaz S, Azhar R, Hameed S. Pattern of liver diseases at Sheikh Zayed Hospital, Lahore. Pak J Med Res. 1995;34:91-94.

9. Trinchet JC, Beaugrand M. Treatment of hepatocellular carcinoma in patients with cirrhosis. J Hepatol. 1997;27:756-765.

10. Stuart KE, Anand AJ, Jenkins RL. Hepatocellular carcinoma in the United States: prognostic features, treatment outcome and survival. Cancer. 1996;77:2217-2222.

11. Mazzaferro V, Regalia E, Doci R, et al. Liver transplantation for the treatment of small hepatocellular carcinoma in patients with cirrhosis. N Engl J Med. 1996;334:693-699.

12. Trojan J, Raedle J, Zeuzem S. Serum tests for diagnosis and follow-up of hepatocellular carcinoma after treatment. Digestion. 1998;59:72-74.

13. Peterson MS, Baron RL, Marsh JW, et al. Pretransplantation surveillance for possible hepatocellular carcinoma in patients with cirrhosis: epidemiology and CT-based tumor detection rate in 430 cases with surgical-pathologic correlation. Radiology. 2000;217:743-749.

14. Yaqoob J, Bari V, Usman MU, et al. The evaluation of hepatocellular carcinoma with biphasic contrast enhanced helical CT scan. J Pak Med Assoc. 2004;54:123-127.

15. Gomaa AI, Khan SA, Leen ELS, et al. Diagnosis of hepatocellular carcinoma. World J Gastroenterol. 2009;15:1301-1314.

16. Murakami T, Mochizuki K, Nakamura H. Imaging evaluation of the cirrhotic liver. Semin Liver Dis. 2001;21:213-224.

17. Koteish A, Thuluvath PJ. Screening for hepatocellular carcinoma. J Vasc Intervent Radiol. 2002;13:S185-S190.

18. Bennett GL, Krinsky GA, Abitbol RJ, et al. Sonographic detection of hepatocellular carcinoma and dysplastic nodules in cirrhosis: correlation of pretransplantation sonography and liver explant pathology in 200 patients. Am J Roentgenol. 2002;179:75-80.

19. Steingruber IE, Mallouhi A, Czermak BV. Pretransplantation evaluation of the cirrhotic liver with explantation correlation: accuracy of CT arterioportography and digital subtraction hepatic angiography in revealing hepatocellular carcinoma. Am J Roentgenol. 2003;181:99-108.

20. Choi BI, Kim TK, Han JK. Power versus conventional color Doppler sonography: comparison in the depiction of vasculature in liver tumors. Radiology. 1996;200:55-58.

21. Fracanzani AL, Burdick L, Borzio M, et al. Contrast-enhanced Doppler ultrasonography in the diagnosis of hepatocellular carcinoma and premalignant lesions in patients with cirrhosis. Hepatology. 2001;34: 1109-1127. 
22. Farooqi JI, Farooqi RJ. Prevalence of hepatocellular carcinoma in patients of liver cirrhosis: an experience in North West Frontier Province (NWFP). J Coll Physicians Surg Pak. 2002;10:54-55.
23. Mumtaz MS, Iqbal R, Umar M, et al. Sero-prevalence of hepatitis $\mathrm{B}$ and $\mathrm{C}$ viruses in hepatocellular carcinoma. J Rawal Med Coll. 2001;5:78-80.

\section{Publish your work in this journal}

The International Journal of General Medicine is an international, peer-reviewed open-access journal that focuses on general and internal medicine, pathogenesis, epidemiology, diagnosis, monitoring and treatment protocols. The journal is characterized by the rapid reporting of reviews, original research and clinical studies across all disease areas.
A key focus is the elucidation of disease processes and management protocols resulting in improved outcomes for the patient. The manuscript management system is completely online and includes a very quick and fair peer-review system. Visit http://www.dovepress.com/ testimonials.php to read real quotes from published authors.

Submit your manuscript here: http://www.dovepress.com/international-journal-of-general-medicine-journal 